Nota de investigación

\title{
Mapeo de la cadena de valor de la tilapia en el estado de Veracruz
}

\author{
Juan Cristóbal Hernández Arzaba ${ }^{1 \S}$ \\ Diego Esteban Platas-Rosado ${ }^{2}$ \\ Alberto Asiain Hoyos ${ }^{2}$ \\ Arturo Pérez Vásquez ${ }^{2}$ \\ Dora Angélica Avalos De la $\mathrm{Cruz}^{3}$ \\ Narciso Ysac Ávila Serrano ${ }^{4}$ \\ ${ }^{1}$ Departamento de Ciencias Económico Administrativas-Universidad Iberoamericana León. Blvd. Jorge \\ Vértiz Campero 1640, Col. Cañada de Alfaro, León, Guanajuato, México. AP. 1-26. CP. 37238. Tel. 477 \\ 7100600, ext. 2619. (cristobal.hernandez@iberoleon.mx). ${ }^{2}$ Colegio de Postgraduados-Campus Veracruz. \\ Carretera Federal Xalapa-Veracruz km 88.5, Tepetates, Manlio Fabio Altamirano, Veracruz, México. CP. \\ 91690. Tel. 229 9207256. (aasiain@colpos.mx; parturo@colpos.mx; dplatas@colpos.mx). ${ }^{3}$ Colegio de \\ Postgraduados-Campus Córdoba. Carretera Federal Córdoba-Veracruz km 348, Manuel León, Amatlán de \\ los Reyes, Veracruz, México. CP. 94946. Tel. 271 7166000. (davalos@ colpos.mx). ${ }^{4}$ Universidad del Mar- \\ Campus Puerto Escondido-Ciudad Universitaria. Carretera Vía Sola de Vega-Puerto Escondido, San Pedro \\ Mixtepec, Juquila, Oaxaca, México. CP. 71980. Tel. 954 5824990. (rebe@ zicatela.umar.mx).
}

${ }^{\S}$ Autor para correspondencia: jcristobal20@ hotmail.com.

\section{Resumen}

El objetivo del presente estudio fue mapear la cadena productiva de tilapia del estado de Veracruz, realizado durante los meses de julio a septiembre de 2016. Para tal efecto, el estudio se realizó en dos etapas. En la primera se efectuó la clasificación de los diferentes actores de la producción de tilapia en los eslabones (materias primas, tipología de productores, proceso y comercialización) de la cadena productiva. En la segunda fueron determinados los siguientes aspectos: tipo de especie, categoría de productores, nivel de producción por categoría, forma de comercialización, presentación del producto, valor agregado, mercados para compraventa y problemáticas para la venta del producto final. Los resultados de la primera etapa del estudio determinaron que los principales insumos son importados y el mercado de mayor importancia es el local. Asimismo, los productores fueron tipificados acorde a su producción (autoconsumo, semi-comercial y comercial) y al mercado que se dirigen (autoconsumo, local y regional). En la segunda etapa de la investigación se encontró que la especie más representativa es la tilapia y su venta es a pie de granja con un valor agregado intrínseco siendo los mercados de compraventa más representativos los de las grandes ciudades. Se concluye que mediante la esquematización de la cadena productiva de la tilapia del estado de Veracruz los principales insumos son importados los cuales deben ser sustituidos por proveeduría y tecnologías nacionales.

Palabras claves: acceso a mercados, acuicultura, competitividad.

Recibido: junio de 2019

Aceptado: julio de 2019 
La tilapia es una de las especies con mayor demanda en el mercado en México. El consumo de esta especie fue cercano a las 200 mil t según la Secretaria de Agricultura, Ganadería, Desarrollo Rural, Pesca y Alimentación (SAGARPA). (Basualdo-Ramírez et al., 2012). Sin embargo, ha existido un déficit debido a que el consumo es derivado de la importación y no de la producción nacional. En este sentido, en el año 2014, se registró una producción aproximada a las 75000 t, la cual posicionó a México como el segundo importador de tilapia a nivel mundial (CONACYT, 2014). HernándezArzaba et al. (2015a) mencionaron que el consumo de pescado en México fue bajo (12 $\mathrm{kg}$ per cápita) en comparación con la media global (20 kg per cápita).

Aunque el consumo per cápita en México es bajo, los productores no alcanzaron a cubrir la demanda del mercado doméstico, estimada en $240000 \mathrm{t}$ por lo que fueron suministrados alrededor de 150000 t de países asiáticos. Este mismo escenario también ocurrió en el estado de Veracruz, en donde fueron consumidas $4000 \mathrm{t}$ de las cuales $2600 \mathrm{t}$ fueron producidas localmente (Hernández-Arzaba et al., 2015b).

El mercado de consumo antes mencionado se comercializa dependiendo de su origen. La tilapia congelada de importación se distribuye a través de grandes comercializadoras (ejemplo: cadenas de restaurantes y supermercados). En contraparte, los productores locales comercializan la tilapia fresca en mercados locales y restaurantes. Actualmente los productores locales de tilapia se encuentran conglomerados debido a los bajos costos de mano de obra y acceso a recursos naturales; sin embargo, son considerados de relevancia baja debido a la escasa tecnología, competitividad y penetración de mercado (Porter, 2003). Ante tal situación, la producción acuícola local de Veracruz tiene un reto comercial y de integración de la cadena de valor. Es por ello que es necesario efectuar el mapeo de la cadena de valor de la tilapia con la finalidad de diagnosticar áreas de oportunidad para la generación de políticas públicas. Por todo lo anterior el objetivo del presente estudio fue mapear la cadena productiva de tilapia del estado de Veracruz.

El área geográfica de cobertura de este estudio se realizó en las regiones fisiográficas denominadas Totonaca, Nautla, Capital, Sotavento, Montañas, Papaloapan y Los Tuxtlas del estado de Veracruz. El estudio fue realizado durante los meses de junio a septiembre de 2016. En la primera etapa se efectuó la clasificación de los diferentes actores de la producción de tilapia en los eslabones de la cadena productiva. Esta cadena fue conformada por los siguientes cuatro eslabones: 1) materias primas; 2) tipología de productores en función de su producción y mercado meta; 3) proceso; y 4) comercialización.

Para tal efecto se obtuvo la información de 814 productores contenidos en el censo acuícola del estado de Veracruz 2010. En la segunda etapa se obtuvieron estadísticas descriptivas en los siguientes aspectos: tipo de especie, categoría de productores, nivel de producción por categoría, forma de comercialización, presentación del producto, valor agregado, mercados para compraventa y problemáticas para la venta del producto final. Los aspectos antes mencionados fueron representados mediante gráficas de pastel en donde se muestra el porcentaje.

\section{Discusión}

Existe una gran área de oportunidad para que los productores locales promuevan y optimicen sus canales de comercialización en sus mercados locales mediante estrategias innovadoras como lo son: los puntos de venta de tilapia viva, venta en tiendas de autoservicio (filetes empacados) y en 
camiones de comida rápida. Lo anterior ya tiene una probada aceptación del consumidor final, a pesar de pagar un sobreprecio por el servicio y la frescura del pescado. Para lograrlo es necesario capacitar y empoderar a los acuicultores, optimizar los programas gubernamentales de apoyo a la industria acuícola y de fomento al consumo de tilapia con valor agregado. Los productores no deben buscar competir con mercados de importación, sino desarrollar un nuevo nicho de mercado para lograr posicionarse. Además, deben incursionar en valor agregado como el ahumado, fileteado, venta de subproductos como la piel (para aplicaciones médicas y de tenería).

Fitzsimmons (2004) postuló en el año 2002, que la tilapia sería el factor clave para la acuacultura en el presente siglo por su fácil manejo y potencial genético; y aceptación en platillo de los grandes mercados globales. Al respecto, México pasó de ser el segundo productor a nivel mundial de tilapia en el año 2002 al $2^{\text {do }}$ lugar como importador de tilapia actualmente, tan solo después de Estados Unidos de América.

\section{Análisis de la cadena de valor}

En la Figura 1 se muestra la esquematización de la cadena productiva de la tilapia en Veracruz mostrando sus eslabones, así como los diferentes mercados a lo que pertenecen. A continuación, se describe cada uno de los eslabones.

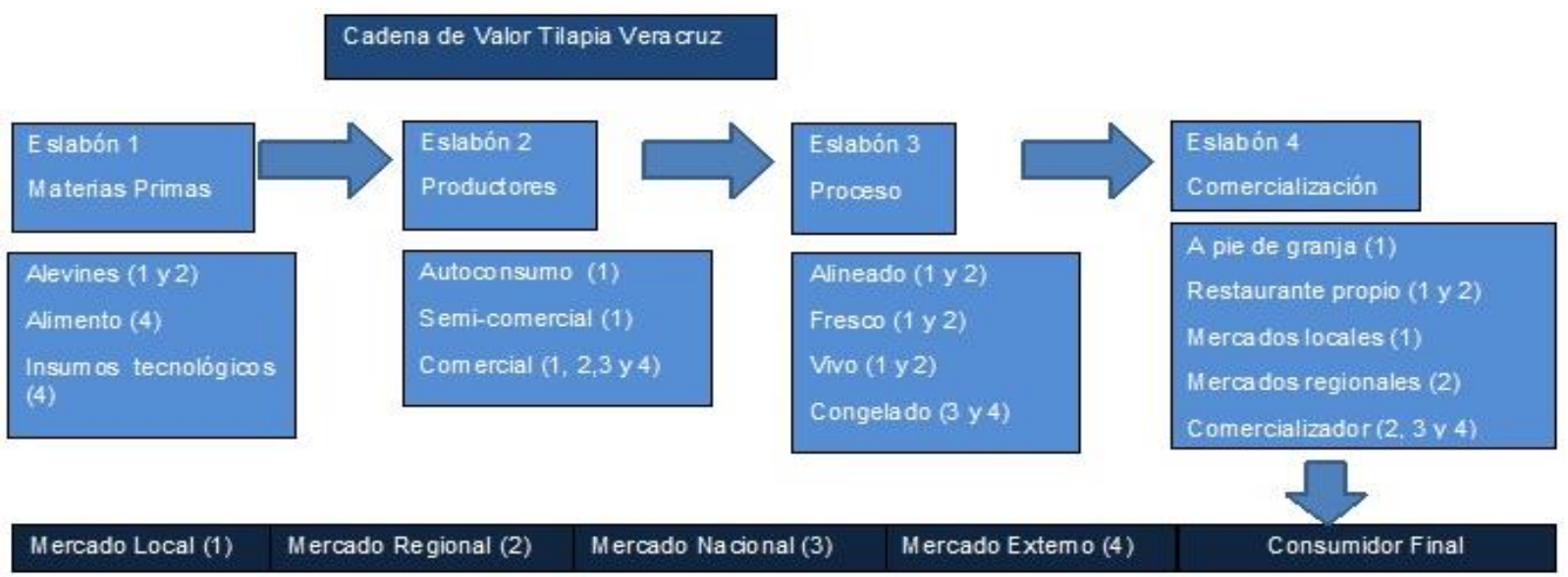

Figura 1. Mapeo de la Cadena de Valor de la Tilapia en Veracruz. Mercados de proveeduría y destino de acuerdo a su origen. Elaboración con datos del ordenamiento acuícola 2010 (Reta y Asiain, 2010).

Eslabón 1. Materias primas: este eslabón se compone por proveeduría, mayoritariamente de importación de insumos (ejemplo: alimento y tecnología). Este efecto puede minimizarse mediante la conformación de redes de proveedores locales que generen dietas basadas en la disponibilidad de materias primas regionales e innovaciones propias. La acuicultura es dependiente de variables de mercados financieros globales (tipo de cambio peso/dólar, tratados de libre comercio, entre otros).

La proveeduría de alevines ha sido desarrollada en Veracruz, pero su oferta aún es muy restringida. Es necesario desarrollar esta cadena de insumos con la selección del mejor material genético haciendo las crías más resistentes y con un mayor porcentaje de carne (CONACYT, 2014). Otra 
de las innovaciones que algunos centros de investigación han venido desarrollando como el Instituto Tecnológico de Boca del Río en Veracruz (ITBOCA), han contribuido en la creación de súper machos que permite evitar el proceso de reversión sexual hasta en un $80 \%$.

Eslabón 2. Tipo de productores: en el Cuadro1 se muestra la tipología de los productores en base a su producción y al mercado meta y a continuación se describe cada categoría:

\section{Autoconsumo}

Incluye a los acuicultores con producción anual total menor a $720 \mathrm{~kg}$ año ${ }^{-1}$; es decir, un promedio de $2 \mathrm{~kg} \mathrm{día}{ }^{-1}$ de consumo; por lo que se infiere que su destino es para los ellos mismos. Estos productores representan 54\% de las granjas de tilapia, más de la mitad de la actividad acuícola más importante en el estado (72.5\% tilapia). Los valores antes mostrados indican que el consumo de tilapia en México se encuentra por debajo de los niveles presentados en países asiáticos, ya que estos tienen un consumo per cápita que rebasa los $20 \mathrm{~kg}$ y en México apenas se logra rebasar la barrera de los $10 \mathrm{~kg}$. La tilapia es la especie con mayor presencia en el estado de Veracruz (Cuadro1).

Cuadro 1. Caracterización de productores de tilapia del estado de Veracruz, con base a la escala de producción.

\begin{tabular}{|c|c|c|c|c|c|c|}
\hline \multirow{2}{*}{ Categoría } & \multirow{2}{*}{ Producción } & \multicolumn{2}{|c|}{ Productores } & \multicolumn{2}{|c|}{ Producción anual } & \multirow{2}{*}{ Mercado destino } \\
\hline & & Número & $(\%)$ & $\mathrm{t}$ & $(\%)$ & \\
\hline Autoconsumo & $\left(<720 \mathrm{~kg}\right.$ año $\left.{ }^{-1}\right)$ & 333 & 53.97 & 74.5 & 4.63 & Autoconsumo \\
\hline Semi-comercial & $\left(720-10000 \mathrm{~kg}\right.$ año- $\left.{ }^{-1}\right)$ & 252 & 40.84 & 712 & 44.26 & Local \\
\hline \multirow[t]{2}{*}{ Comercial } & $\left(>10 \mathrm{t}\right.$ año $\left.{ }^{-1}\right)$ & 32 & 5.19 & 822 & 51.1 & Regional/estatal \\
\hline & Total & 617 & 100 & 1608.5 & 100 & \\
\hline
\end{tabular}

Elaboración con datos del ordenamiento acuícola del estado de Veracruz 2010.

La especie más representativa de la acuicultura en Veracruz es la tilapia ya que representa casi $75 \%$ de las granjas, seguido de la trucha con poco más de $20 \%$, con casi $4 \%$ se presentan los peces de ornato y otras especies tales como el ostión, caracol y langostino (Figura 2a).

Los productores de autoconsumo representan más de 50\%, pero aportan $4.6 \%$ de la producción total anual. Este grupo de productores contribuyen a la seguridad alimentaria, aunque no la resuelven, dado que no crean dinámicas microeconómicas en su área de influencia. Por ello, es importante desarrollar políticas públicas para fomentar el apoyo de dicha actividad para la construcción de una pirámide de seguridad alimentaria, una proveeduría alterna de proteína animal, una dieta balanceada, y una actividad primaria detonadora en economías locales. Esto puede efectuarse mediante la dotación de programas de información, capacitación extensionismo a los productores con el objetivo de hacer más eficientes las granjas de autoconsumo con los recursos disponibles y así contar con excedentes que se destinen al mercado. 


\section{Semi-comercial}

Este tipo de cultivadores son los predominantes en la producción de tilapia en Veracruz, se encuentran casi en equilibrio de acuerdo con el porcentaje de productores y la producción que aportan; presentando una razón de 1.08 (número de granjas-capacidad productiva) tal como se muestra en el Cuadro 2. Adicionalmente, esta categoría produce excedentes que pueden ser comercializados y que desarrollan dinámicas económicas dado que se encuentran en la frontera de posibilidades de producción, (FPP). También se observó que esta categoría tiene acceso a mercados locales, se auto-emplea el dueño de la granja y al menos un jornalero con tendencia a la participación familiar.

Cuadro 2. Razón financiera de los productores de tilapia

\begin{tabular}{lccc}
\hline Variable & Productores $(\%)$ & Producción $(\%)$ & Razón \\
\hline Autoconsumo & 53.97 & 4.63 & 0.09 \\
Semi-comercial & 40.84 & 44.26 & 1.08 \\
Comercial & 5.19 & 51.1 & 9.85 \\
\hline
\end{tabular}

Elaboración con datos del ordenamiento acuícola del estado de Veracruz 2010.

\section{Comercial}

Esta categoría alcanza una producción de tilapia mayor a 10 ton anuales y se clasifica dentro del área de las MIPYMES. Representan un grupo pequeño de productores, tan solo el $5.19 \%$ del total (Figura 2b). Sin embargo, su capacidad de producción es importante debido que con 5\% de granjas aportan más de la mitad de la producción anual (51.1\%) tal como se observa en la Figura 2c.

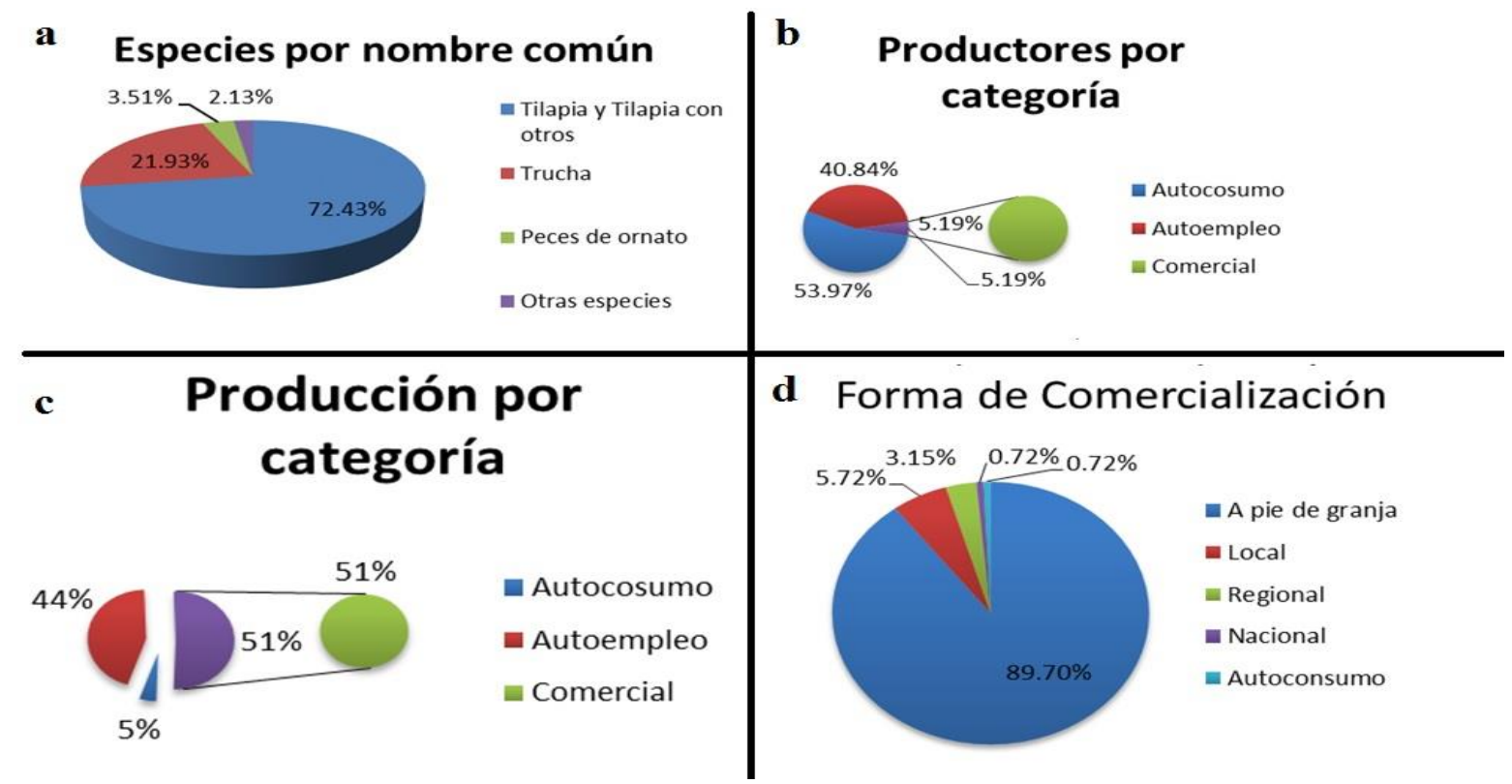

Figura 2. a) especies por nombre común; b) productores por categoría; c) producción por categoría; y d) forma de comercialización. Elaboración con datos del ordenamiento acuícola del estado de Veracruz (2010). 
El sector comercial tiene bien definido que la producción de tilapia es un negocio rentable, su mercado es regional, estatal y en pocos casos interestatales o con fines de exportación. Para el fortalecimiento de esta categoría debe consolidarse en el corto y mediano plazo, desarrollando un mercado sin competir con las importaciones asiáticas. Posteriormente, diversificarse y dar valor agregado para ser competitiva en el mediano y largo plazo. A largo plazo deberá considerar la opción de exportar y cubrir la demanda nacional de tilapia, que permita iniciar un mercado competitivo por calidad y frescura.

Debido a la demanda creciente de pescado, esta categoría puede buscar alianzas estratégicas con la categoría semi-comercial para incrementar su capacidad productiva. La categoría comercial puede proveer técnicas y requisitos de estandarización del producto deseado al sector semi-comercial, así como ofertar compra por volumen. En contraparte, el sector de autoconsumo puede obtener conocimientos para un mejor manejo y tecnificación de la granja aspirando a potencializar su producción y comercialización.

\section{Eslabón 3 y 4. Proceso y comercialización}

En la Figura 2d, se observa que la comercialización a pie de granja predomina (89.7\%) sobre el resto de las formas de venta. La participación en el mercado local es representativa (95.42\%) siendo casi nula la participación en mercados regionales y nacionales. Los datos anteriores pueden ser derivados por la falta de infraestructura y mantenimiento de la existente, costos de transporte y la falta de habilidad en gestión empresarial.

Con base en la información presentada en la Figura 3a se observa que el mayor consumo se basa a pie de granja: vivo o fresco. Este tipo de presentación puede ser considerado como un valor agregado que presenta calidad y frescura del producto. Sin embargo, la nula expansión comercial puede deberse a la falta de inversión e infraestructura en cadenas de frío.

Respecto a la agregación de valor al producto (Figura 3b), el $82.34 \%$ de los productores no dan valor agregado. Este tipo de respuesta justifica la puesta en marcha de prácticas (capacitación en la presentación del producto, otras formas de venta, entre otros) para incrementar el ingreso de los acuicultores. Considerando que el promedio de precio de la tilapia viva y alineada es de $\$ 41.48 \mathrm{~kg}$; los tipos de valor agregado son en primer término cuando el acuicultor transforma su producción en un platillo en su restaurante, en forma de filetes sin/con empaque, y ahumado.

En la Figura 3c se muestra que los mercados preponderantes para venta son Xico (21\%), Alvarado $(8 \%)$, Veracruz $(8 \%)$, Xalapa (8\%) y Ciudad de México $(7 \%)$, que juntos representan más de la mitad del mercado $(51 \%)$. Esto puede deberse a que las cuatro primeras ciudades forman parte de las dos zonas metropolitanas más grandes del estado de Veracruz y en el caso de la Ciudad de México, es el mercado de más alto consumo del país.

Por otro lado, en la Figura 3d, los mercados de compra están representados por Xalapa (11.57\%), Cd. Cardel (7.65\%), Cuitláhuac (6.47\%), Córdoba (6.08\%), Veracruz (5.69\%), Xico (5.29\%) y Orizaba (4.51\%), sumando casi la mitad de este mercado (47.26\%). Lo anterior, puede deberse a que dichas poblaciones pertenecen a tres de las zonas metropolitanas más grandes del estado de Veracruz. 


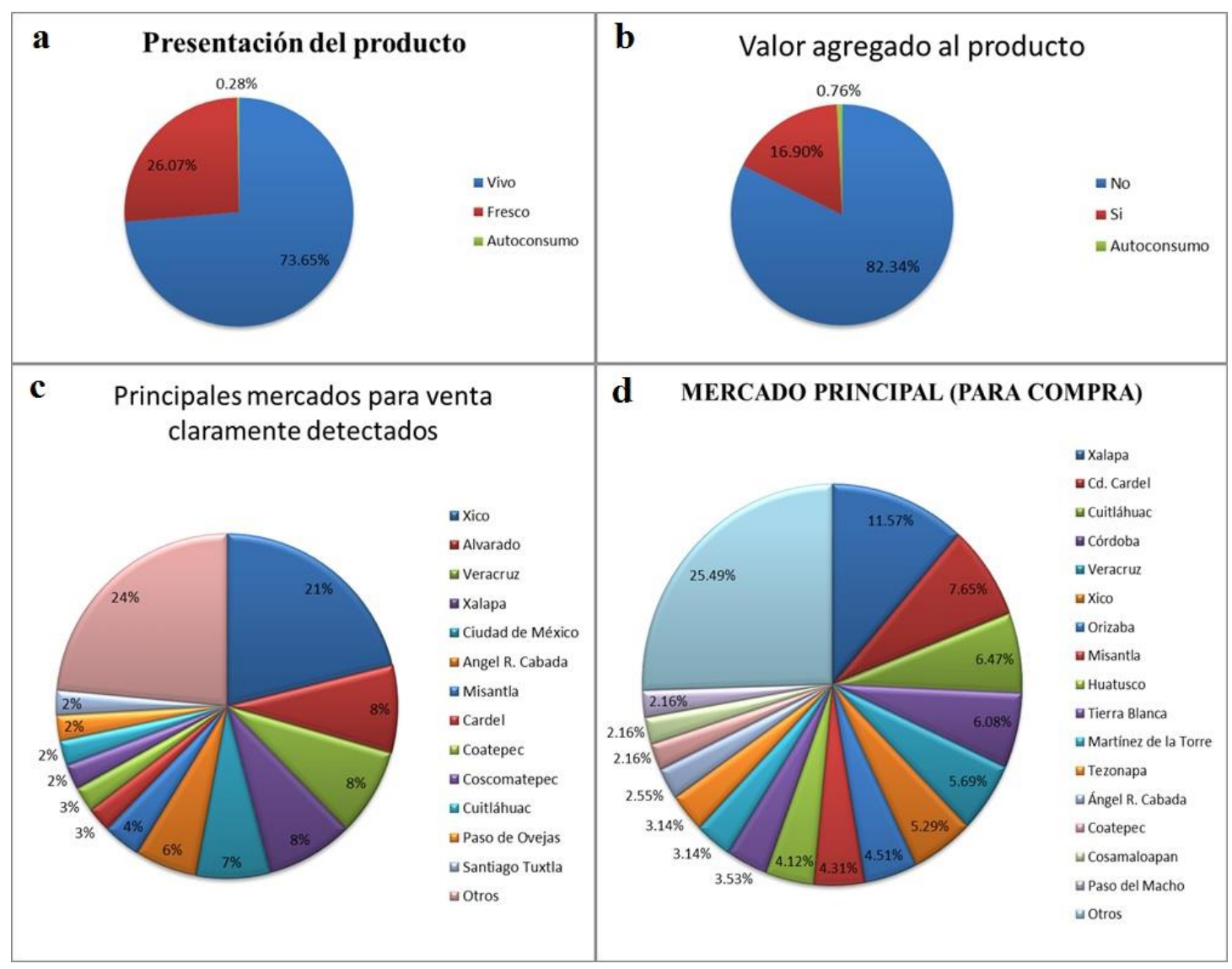

Figura 3. a) presentación del producto; b) valor agregado del producto; c) principales mercados para venta claramente detectados; $y$ d) mercado principal (para compra).

En la Figura 4a, se observó que más de $91 \%$ de los entrevistados no tienen problema en la comercialización por lo que se asume que la tilapia es un producto que se vende por sí solo, pero la falta de acceso a mercados debe ser empresa del sector gubernamental, mejorando y creando infraestructura.

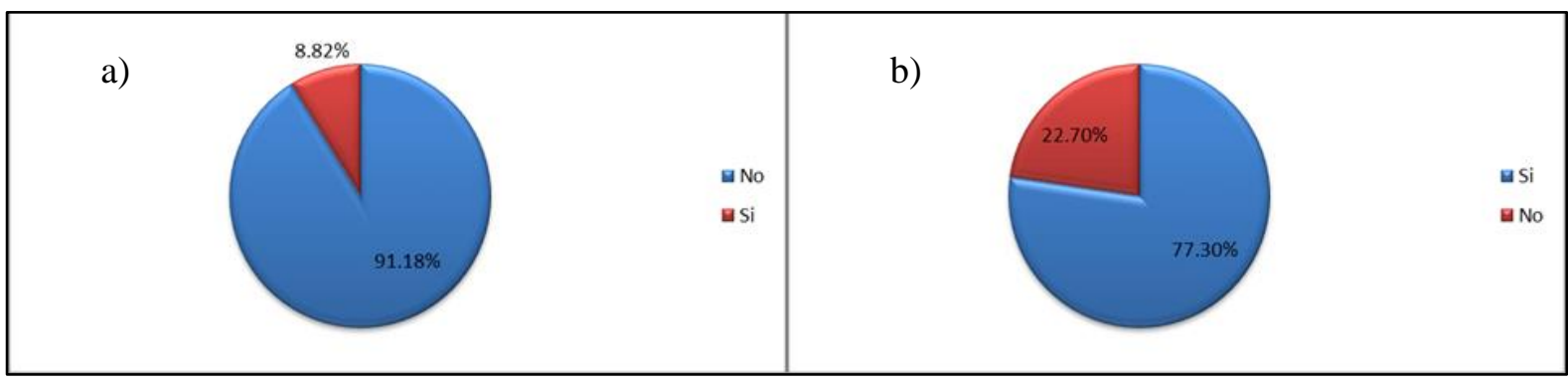

Figura 4. a) ¿tiene problemas para vender su producto?; y b) ¿le interesaría unirse a una empresa integradora?

Finalmente, en la Figura 4b se observó que 77.3\% de los acuicultores tiene interés en pertenecer a una empresa integradora, esto puede deberse a los beneficios de las uniones de productores (por ejemplo: compartir conocimientos de manejo y producción, comercializar por volumen y bajar costos de insumos por compras al mayoreo, entre otros). 
Sin embargo, el restante $22.70 \%$ respondió no, esto puede ser debido a la corrupción de los líderes, el mal funcionamiento de los sistemas-producto, así como por la idiosincrasia y cultura del productor.

\section{Conclusiones}

En la mediante la esquematización de la cadena productiva de la tilapia del estado de Veracruz los principales insumos son importados, los cuales deben ser sustituidos por proveedurías y tecnologías nacionales. De la misma manera, la tipología de los acuicultores en función de su producción mostró que estos deben ser capacitados en el manejo y comercialización de sus productos. También que los productores no tienen una diversificación del valor agregado de sus productos, inhibiendo su acceso al mercado local y regional. Los acuicultores no promueven el consumo de sus propios productos, mientras que el gobierno realiza una propaganda somera. Por último, se recomienda que los apoyos gubernamentales sean canalizados a los cultivos acuícolas con mayor predominancia y a los productores comerciales.

\section{Literatura citada}

CONACYT. 2014. Consejo Nacional de Ciencia y Tecnología. Agencia Informativa. Diseñan modelo integral para la producción de tilapias. http://www.conacytprensa.mx/index.php/ ciencia/mundo-vivo/591-produccion-de-tilapias.

Basualdo-Ramírez, L. J.; Jiménez-Guzmán, F.; Jiménez-Saavedra, A. C.; Macal-Niño, F. J.; Basualdo-Ramírez, E. A. M.; Montaño-Aguilar, D. M. A. y Gutiérrez, U. E. 2012. Criterios técnicos y económicos para la producción sustentable de tilapia en México. https://www.conapesca.gob.mx/work/sites/cona/dgof/publicaciones/CriteriosTecnicosEco nomicosTilapiaEnMexico.pdf.

Fitzsimmons, K. 2004. Producción y mercado internacional de tilapia. In: International symposia on tilapia in aquaculture. Septiembre. Manila, Filipinas. 133-149 pp.

Hernández-Arzaba, J. C.; Platas-Rosado, D. E.; González-Reynoso, L.; Preza-Lagunes, L. y Torres-Tadeo, C. M. 2015a. Mapeo en la cadena de comercialización/consumo de tilapia en Veracruz. In: I Reunión Internacional Científica y Tecnológica. Veracruz, México. 107-115 pp.

Hernández-Arzaba, J. C.; Platas-Rosado, D. E.; González-Reynoso, L.; Preza-Lagunes, L. y Torres-Tadeo, C. M. 2015b. Evidenciando el desarrollo de la acuacultura. Mapeo en la cadena de valor de tilapia en México. En P. Pérez (Presidencia), XLII Reunión Científica de la Asociación Mexicana para la Producción Animal y Seguridad Alimentaria A. C. Reunión llevada a cabo en Montecillo, Texcoco, Estado de México.

Porter, M. E. 2003. The economic performance of regions. Regional Studies. 37(6-7):549-578. doi: 10.1080/0034340032000108688.

Reta-Mendiola, J. L. y Asiain-Hoyos, A. 2010. Ordenamiento acuícola del estado de Veracruz. SAGARPA/CONAPESCA/Colegio de Postgraduados. 26 p. 